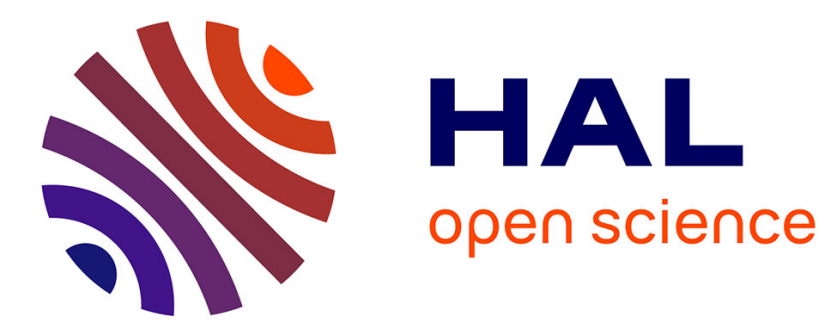

\title{
Experimental study of hot spots in gold/glass nanocomposites films by photoemission electron microscopy
}

Chawki Awada, Grégory Barbillon, Fabrice Charra, Ludovic Douillard, Jean-Jacques Greffet

\section{To cite this version:}

Chawki Awada, Grégory Barbillon, Fabrice Charra, Ludovic Douillard, Jean-Jacques Greffet. Experimental study of hot spots in gold/glass nanocomposites films by photoemission electron microscopy. Physical Review B: Condensed Matter and Materials Physics (1998-2015), 2012, 85, pp.045438. 10.1103/PhysRevB.85.045438 . hal-00785293

\section{HAL Id: hal-00785293 \\ https://hal-iogs.archives-ouvertes.fr/hal-00785293}

Submitted on 5 Feb 2013

HAL is a multi-disciplinary open access archive for the deposit and dissemination of scientific research documents, whether they are published or not. The documents may come from teaching and research institutions in France or abroad, or from public or private research centers.
L'archive ouverte pluridisciplinaire HAL, est destinée au dépôt et à la diffusion de documents scientifiques de niveau recherche, publiés ou non, émanant des établissements d'enseignement et de recherche français ou étrangers, des laboratoires publics ou privés. 


\title{
Experimental study of hot spots in gold/glass nanocomposite films by photoemission electron microscopy
}

\author{
C. Awada, ${ }^{1}$ G. Barbillon, ${ }^{2}$ F. Charra, ${ }^{1}$ L. Douillard, ${ }^{1}$ and J.-J. Greffet ${ }^{2, *}$ \\ ${ }^{1}$ Service de Physique et Chimie des Surfaces et Interfaces, CEA, IRAMIS, F-91191 Gif sur Yvette, France \\ ${ }^{2}$ Laboratoire Charles Fabry de l'Institut d'Optique CNRS UMR 8501, Université Paris-Sud, Campus Polytechnique, RD-128, F-91127 \\ Palaiseau cedex, France
}

(Received 10 December 2011; published 24 January 2012)

\begin{abstract}
In this paper, an experimental study of hot spots in gold/dielectric films using photoemission electron microscopy is reported. This technique allows a characterization of the statistical optical properties with unprecedented accuracy in the 800- to 1040-nm range. Theoretical predictions of the scaling theory on the number and intensity wavelength dependences of hot spots in the near-infrared are confirmed. Statistical properties of the intensity distribution, spectral behavior, and spatial localization of the hot spots are reported.
\end{abstract}

DOI: 10.1103/PhysRevB.85.045438

PACS number(s): 78.67.-n, 78.68.+m

\section{INTRODUCTION}

Disordered metallodielectric systems have attracted considerable attention for their unique optical and electrical properties. ${ }^{1-3}$ In particular, semicontinuous metallic films on dielectric surfaces present optical properties that differ markedly from isolated particles or continuous films. ${ }^{1}$ Among these unique properties, let us point out a very large absorption over a broad spectrum and giant fluctuations of the absorption. A large number of theoretical studies ${ }^{2,3}$ have finally converged to a simple picture to explain these properties. Random systems sustain localized modes due to coherent multiple scattering in the random system. In addition, these localized modes can be further enhanced locally due to the presence of tips or nanogaps. In the visible, particle plasmon resonances play a key role, whereas in the IR, the enhancement is only due to resonant excitation of modes of the disordered system. The combination of these mechanisms produce highly localized hot spots (HS) on the nanometer scale. ${ }^{4-8}$ A remarkable property of these localized modes is that they are observed for any frequency as predicted by numerical simulations and scaling theory. In addition, simple frequency dependence of the density of HS and their intensity are predicted by scaling theory in the infrared. ${ }^{3,6}$ More recently, it has been predicted that delocalized modes consisting of several HS can exist. ${ }^{9}$

Let us stress that the theoretical models developed so far have considered systems with a total size smaller than the wavelength so that retardation effects are not included. Hence, experiments with high spatial resolution are needed to confirm the models. The first generation of experiments dealt with ensemble absorption measurements, which clearly demonstrated the spectral broadening in the semicontinuous regime, ${ }^{1-3}$ a behavior very different from the absorption spectra of isolated nanoparticles or continuous films. Images of the localized enhancements were reported using near-field microscopy by several groups, ${ }^{10-14}$ confirming the existence of localized spots on the nanometer scale. The existence of delocalized modes has been supported by the observation of higher-order mode correlations of intensity fluctuations ${ }^{15}$ and more recently by a detailed study of the statistical distribution of fluorescent-molecule lifetimes in the visible range. ${ }^{16}$ Despite all these contributions, several questions are still open: (i) As near-field microscopy is easier to operate in the visible range, no data are available in the IR part of the spectrum for wavelengths larger than $800 \mathrm{~nm}$. This is a domain where HS are genuine disorder effects with remarkable properties predicted by the scaling theory. (ii) It is difficult to fully assess the perturbation of the tip on the system. (iii) The spatial distribution of HS over the surface has not been investigated in detail. Although numerical simulations suggest that the HS should be preferentially localized on some specific sites, no experimental evidence is available to confirm this prediction. To address these issues, we report the first study of HS in gold/glass nanocomposite films in the near-infrared range with a photoemission electron microscope (PEEM).

\section{EXPERIMENTAL DETAILS}

The PEEM technique [Fig. 1(a)] gives access to full-field images of the near optical field in the near-infrared range $(800-1040 \mathrm{~nm})$ with a spatial resolution of $20 \mathrm{~nm}$. Given the large amount of data, it is possible to study with unprecedented accuracy the statistical optical properties of metal dielectric films under light excitation. The samples are illuminated by a femtosecond pulsed laser (Ti:sapphire ultrafast oscillator Chameleon Ultra II, Coherent Inc., repetition rate $80 \mathrm{MHz}$, hyperbolic-secant-squared pulse, pulse width $140 \pm 20 \mathrm{fs}$ ). The polarization of the laser light can be controlled by a half-waveplate. The incidence angle is $\alpha=73^{\circ}$ with respect to the surface normal. The experimental conditions are optimized to keep constant the intensity, the pulse width and the focus position of the incident beam during data acquisition. The PEEM instrument used is a commercial model [Elmitec $\mathrm{GmbH}$ low energy electron emission (LEEM)/PEEM III], which operates under ultrahigh vacuum conditions. On a flat surface, lateral spatial resolutions of 10 and $20 \mathrm{~nm}$ are routinely achieved in LEEM- and PEEM-imaging modes, respectively.

In contrast to local probe techniques, the imaging principle makes use of the electron emission at HS through a multiphoton light absorption process. Indeed, the commonly accepted underlying mechanism for the increase of the photoemission yield observed close to a plasmon energy is an $n$-step cascade photon absorption process (in contrast to direct vertical transitions via simultaneous absorption of $n$ photons). ${ }^{17}$ The additional momentum transfer associated with indirect 


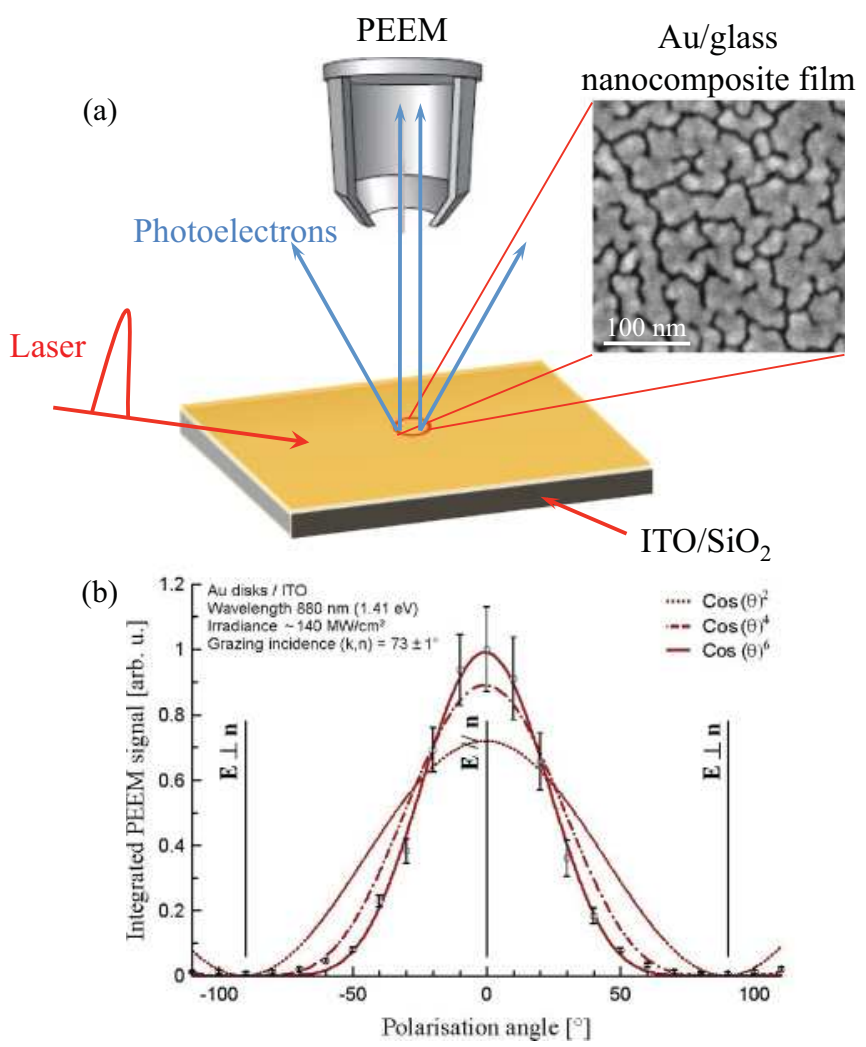

FIG. 1. (Color online) (a) Principle scheme of the photoemission electron microscopy experiment and SEM image of Au/glass nanocomposite film with a gold filling factor (apparent surface coverage) of $f=0.76$, and (b) polarization dependence of the electron yield of Au disks measured by photoemission. ${ }^{18}$ transition is presumably due to local defects, i.e., stacking faults, grain boundaries, impurities acting as momentum sources. Experimentally, an apparent 3-photon photoemission regime is observed in the investigated wavelength window (800-1040 nm). We observe a nonlinearity order $n=3$ by measuring the dependence of the electron yield of Au disks [see Fig. 1(b)] on the polarization of the incident beam. ${ }^{18}$ For the $\mathrm{Au}$ particles excited at $880 \mathrm{~nm}(1.41 \mathrm{eV})$, the data fitting with a $(\cos \theta)^{2 n}$ function points to a $n=3$ apparent multiphoton process lower than the $n=4$ order expected from a work function of $\mathrm{Au}$ film either amorphous ${ }^{19} 4.7 \mathrm{eV}$ (present case) or crystalline $5.3 \mathrm{eV}$. This lower than expected value is currently interpreted on a basis of plasmon assisted multiphoton emission. ${ }^{20-22}$

In PEEM-imaging mode, the measured brightness in a given image area is proportional to the electron emission flux from this area. Integrated PEEM signal is computed in two steps: (i) CCD background substraction, and (ii) integration of the pixel values over a representative image area. Further technical details can be found in Refs. 18, 23, and 24. The substrate samples correspond to polished high-quality float glass substrates coated with a thin indium tin oxide (ITO) layer. Surface resistivity of the ITO coating is lower than $50 \mathrm{ohms} / \mathrm{sq}$, its thickness corresponds to about $40 \mathrm{~nm}$ (CEC050P, Präzisions Glas \& Optik GmbH). Prior to Au deposition, glass substrates are cleaned with ethanol and acetone successively. Au deposition is carried out in a vacuum chamber equipped with a thermal evaporator based on the ohmic heating up to fusion of gold shot (99.9999\% Strem Chemicals inc.). The mass of deposited metal is monitored in situ by a water cooled quartz microbalance. Typical evaporation parameters are a flux of $2 \dot{\mathrm{A}} / \mathrm{min}$ at a substrate temperature slightly above

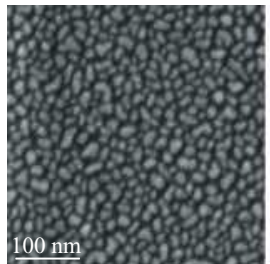

$\mathrm{Au} 4 \mathrm{~nm}, \mathrm{f}=0.53$

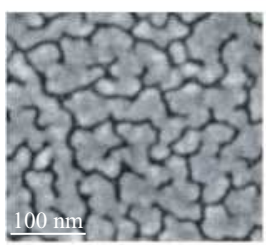

Au $8 \mathrm{~nm}, \mathrm{f}=0.76$

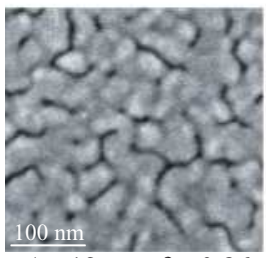

$\mathrm{Au} 12 \mathrm{~nm}, \mathrm{f}=0.86$
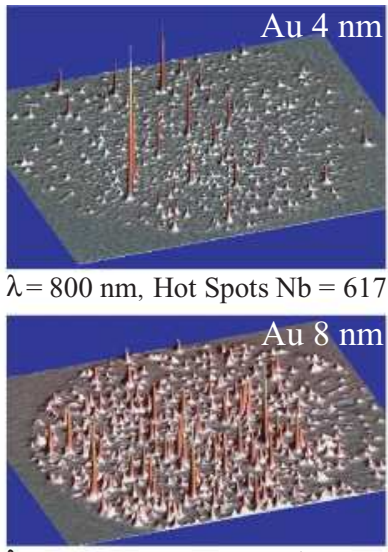

$\lambda=800 \mathrm{~nm}$, Hot Spots $\mathrm{Nb}=426$

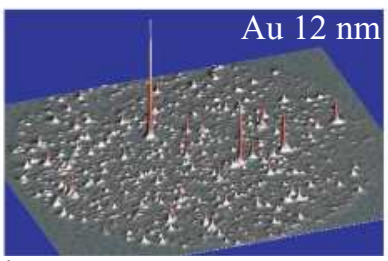

$\lambda=800 \mathrm{~nm}$, Hot Spots $\mathrm{Nb}=672$

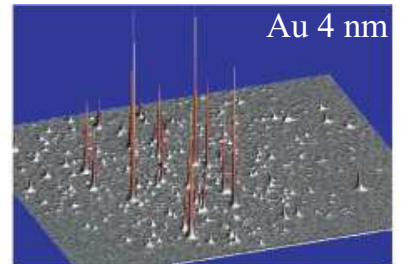

$\lambda=930 \mathrm{~nm}$, Hot Spots $\mathrm{Nb}=453$

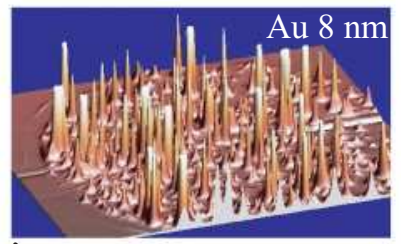

$\lambda=930 \mathrm{~nm}$, Hot Spots $\mathrm{Nb}=250$

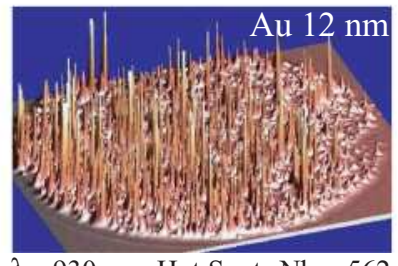

$\lambda=930 \mathrm{~nm}$, Hot Spots $\mathrm{Nb}=562$

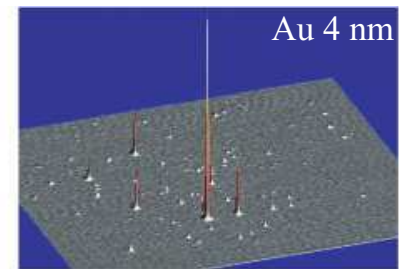

$\lambda=970 \mathrm{~nm}$, Hot Spots $\mathrm{Nb}=402$

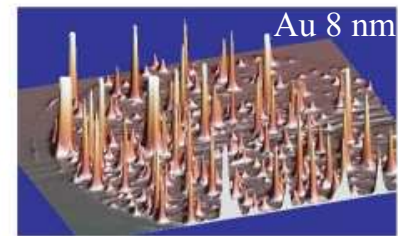

$\lambda=970 \mathrm{~nm}$, Hot Spots $\mathrm{Nb}=198$

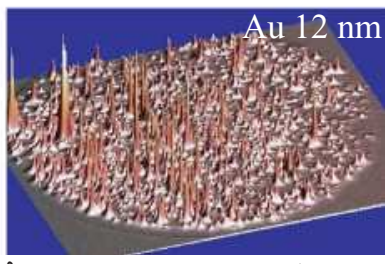

$\lambda=970 \mathrm{~nm}$, Hot Spots $\mathrm{Nb}=485$

FIG. 2. (Color online) Left column, SEM images of the gold/glass films for $4 \mathrm{~nm}(f=0.53), 8 \mathrm{~nm}(f=0.76)$, and $12 \mathrm{~nm}(f=0.86)$. Right, 3D PEEM images corresponding to gold/glass films for three different wavelengths: for $4 \mathrm{~nm}$, the $Z$ axis is zoomed $\times 3$; for $8 \mathrm{~nm}, \times 1$; for $12 \mathrm{~nm}, \times 1.5$ at $800 \mathrm{~nm}$ and $\times 1$ at 930 and $970 \mathrm{~nm}$. For each PEEM image, the excitation wavelength and the number of HS are indicated. The diameters of the PEEM images field are $15 \mu \mathrm{m}(4 \mathrm{~nm}), 10 \mu \mathrm{m}(8 \mathrm{~nm})$, and $13 \mu \mathrm{m}(12 \mathrm{~nm})$. 


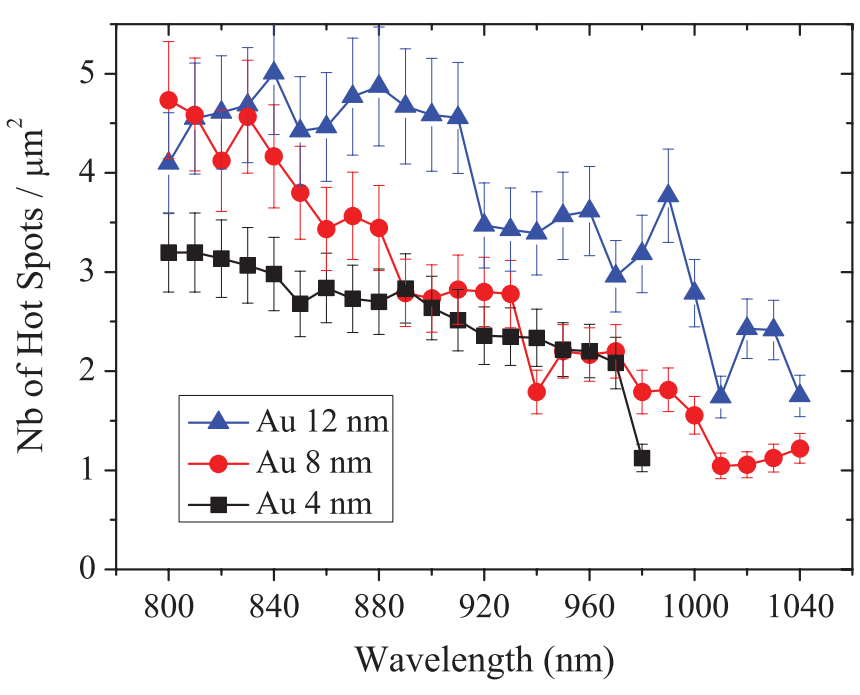

FIG. 3. (Color online) Density of HS versus $\lambda$ for three filling factors in the near-infrared range: black squares corresponding to $f=0.53$, red circles to $f=0.76$, and blue triangles to $f=0.86$.

room temperature (no sample heating). The base pressure during evaporation is in the low $10^{-5}$ mbar and the angle of incidence of the Au beam is close to the surface normal. A set of three samples has been prepared corresponding to equivalent deposited Au thicknesses of 4,8 , and $12 \mathrm{~nm}$, respectively. The corresponding filling factors are $f=0.53$, 0.76 , and 0.86 , respectively, as determined by SEM. Typical SEM images of the gold/glass films are presented in the left column of Fig. 2. It is seen that the system consists of isolated gold islands for $f=0.53$, of interconnected gold clusters for $f=0.76$, and that the system tends to a continuous metallic film with voids for $f=0.86$. It is important to introduce the percolation threshold $f_{p}$, which takes the value $0.68^{25}$ for a monodisperse ensemble of disks. The optical properties of the films are expected to differ markedly for these three regimes as discussed in Refs. 26- 29.

\section{RESULTS AND DISCUSSION}

The PEEM images of the three samples excited at three different wavelengths are shown in Fig. 2 . We plot the cubic root of the PEEM signal in order to display the intensity. The signal was not normalized so that it is given in arbitrary units. At first glance, we observe a large number of HS of various intensities with a structure very similar to previously reported numerical simulations. The remarkable resolution of the instrument and the intrinsic intensity threshold due to the three-photon absorption process allows imaging the HS without background noise. We now turn to a quantitative analysis of the data. For a given filling factor (same line in Fig. 2), the number of HS decreases when the excitation wavelength increases. We also note that the peak intensity increases. This qualitative observation is quantified in Fig. 3, where we have plotted the density of HS per $\mu \mathrm{m}^{2}$ as a function of the excitation wavelength for the three filling factors investigated. For each sample, we observe a linear decay of the HS density for wavelengths larger than $880 \mathrm{~nm}$. This is in agreement with the prediction of the scaling theory ${ }^{3,6,13}$ valid in the IR regime for $\omega_{p} \gg \omega$. Upon comparison of the three samples, we also note that the density of HS increases when the filling factor $f$ also increases.

A more complete characterization can be obtained by studying the intensity distribution. We have plotted in Fig. 4 the histograms of the intensities observed in Fig. 2. The distribution of intensity probability changes significantly when varying the filling fraction. To analyze the data, we use the log-normal distribution given by

$$
P(I)=\frac{A}{I \sigma \sqrt{2 \pi}} \exp \left[-\left(\frac{(\ln I-<\ln I>)^{2}}{2 \sigma^{2}}\right)\right],
$$

where $\langle\ln I\rangle$ is the average value of the logarithm of the intensity $I, \sigma$ is the standard deviation in terms of $\ln I$, and $A$ is a constant. Remarkably, the log-normal distribution (red curve) fits the data for all values of the filling fraction and all incident wavelengths as seen in Fig. 4. It is clearly seen that for $f=0.53$, in the nonconnected island regime, low intensities are the most frequent. By contrast, the intensity distribution is centered around larger values as the filling fraction approaches or exceeds the percolation threshold. It is also clear that the distribution broadens significantly as already reported in Ref. 15 by studying the higher moments of the distribution. An important result of our study is the observation of very large values of the intensity at $f=0.76$ and for wavelengths larger than $900 \mathrm{~nm}$ not explored previously. Hence, the intensity distribution displays a long tail. Note that for experimental reasons, the measurement dynamic range is saturated for the sample close to the percolation threshold $f=0.76$. Hence, the peak at a value of 16 is thus representative of all the intensities larger than 16. These values correspond to the large truncated peaks observed in Fig. 2 for 8-nm film thickness and 930- or 970-nm excitation wavelength.

We now address the issue of the spatial distribution of the hot spots. It has been suggested by numerical simulations that HS appear preferentially at some particular locations (hereafter called sites) such as tips or nanogaps. An example of such a correlation between the position of a HS and the existence of a nanogap has been shown in Ref. 30. If this correlation is a general feature, resonances should be observed at the same site for different frequencies. On the contrary, if the presence of a HS is only due to the existence of a mode of the disordered system independently of some specific feature of a particular realization, no correlation of the resonance positions for different frequencies are expected. To investigate this issue, we have studied the location of the resonances observed in a given area $A$ for several frequencies in the range of 800-1040 nm. From the analysis of the spectrum of all the pixels displaying at least one HS in a given area $A_{\mathrm{Sa}}$ of the sample, we have identified $N$ different resonances distributed among $M^{\prime}$ different sites. Hereafter, we call pixel a group of pixels of area $A_{\mathrm{pi}}$ chosen to match the spatial resolution of the instrument. The total number of independent pixels in the sample is thus $M=A_{\mathrm{Sa}} / A_{\mathrm{pi}}$. We have found that some pixels can host up to six resonances in the spectral interval but most of the pixels never host a resonance. A characteristic example of a PEEM resonance spectrum recorded at one fixed site is plotted in Fig. 5. At the considered site, four distinct resonances with an average full-width at half-maximum of $\sim 18 \mathrm{~nm}$ are observed in the 780- to $920-\mathrm{nm}$ wavelength range. 


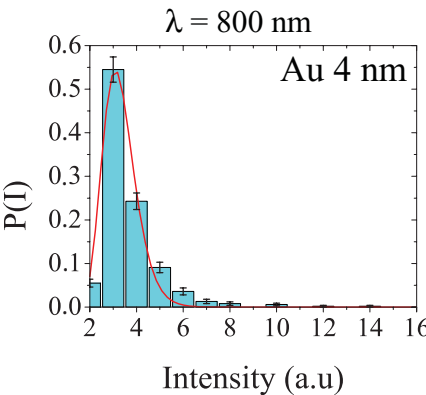

(a)

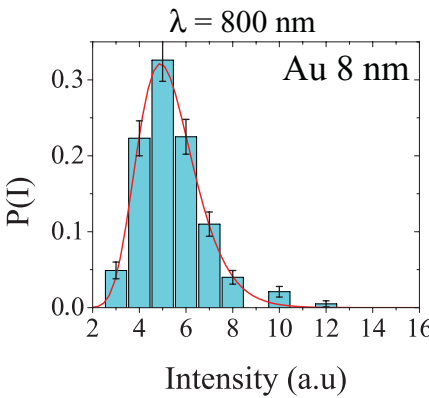

(d)

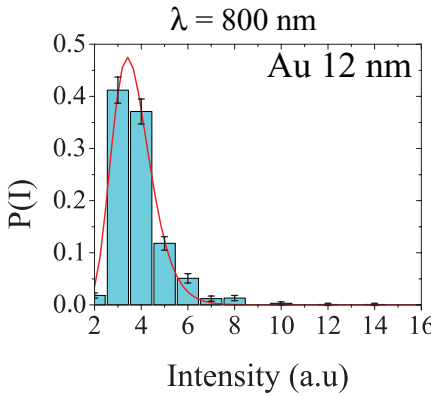

(g)

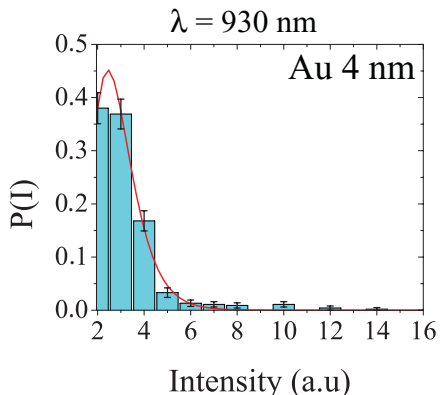

(b)

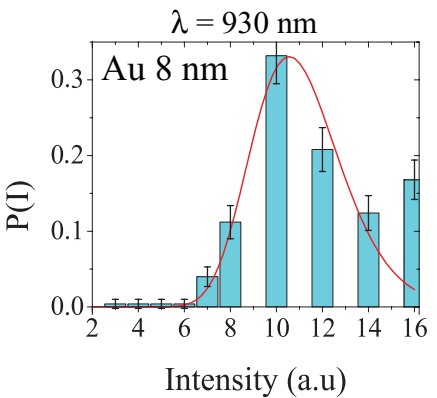

(e)

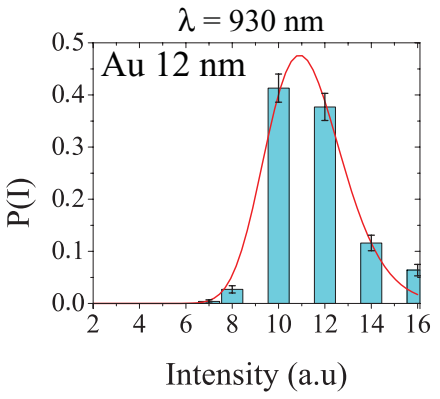

(h)

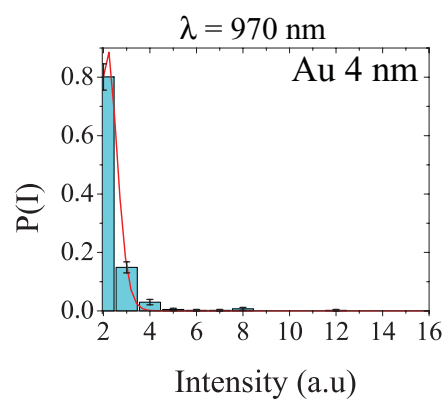

(c)

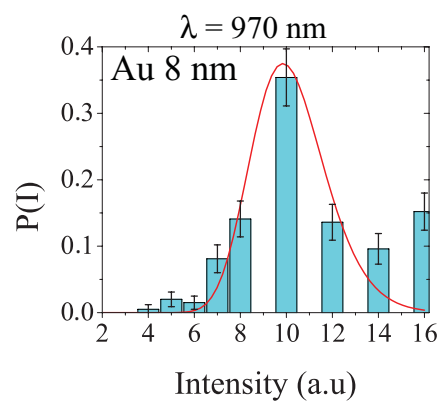

(f)

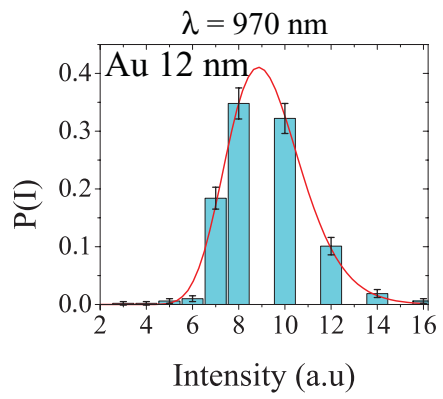

(i)

FIG. 4. (Color online) Log-normal distribution $P(I)$ of intensity probability at different wavelengths for three filling factors: the blue bars represent the experimental data, and in red, the log-normal data fit. On each distribution, the filling factor and the incident wavelength are indicated. Error bars correspond to the square root of the standard deviation of the histogram bar populations.

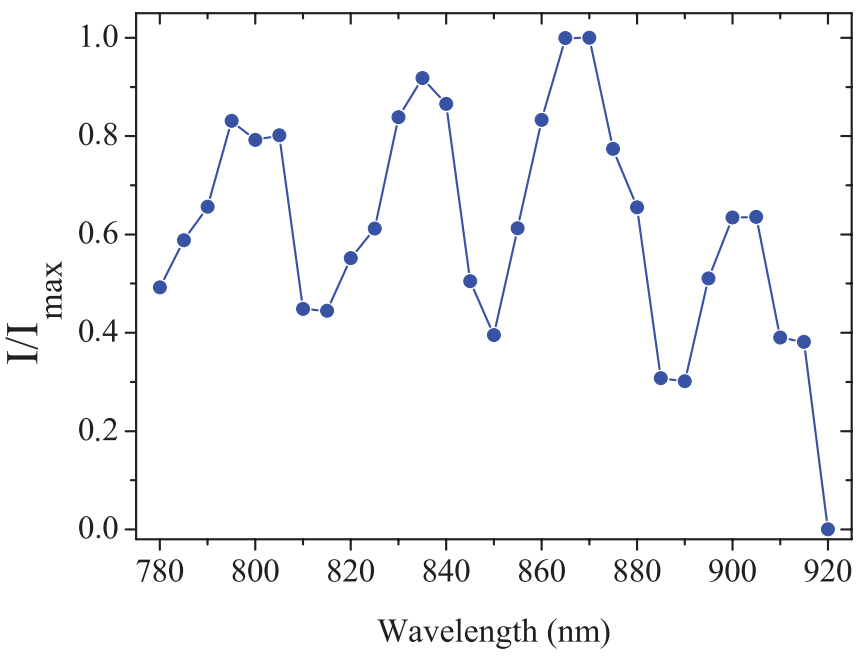

FIG. 5. (Color online) Characteristic high-resolution PEEM intensity spectrum recorded at one fixed site.
We present in Fig. 6 a histogram classifying the pixels according to their number of resonances in the range 800 $1040 \mathrm{~nm}$. Figures 6(a), 6(c), and 6(e) represent the probability of having $N$ resonances for an arbitrary pixel in the observed area. The red curve shows the probability assuming a Poisson process such as the HS would be equally distributed over all possible pixels on the surface. The probability is then given by

$$
P(N=k)=\frac{\left(\gamma A_{\mathrm{Sa}}\right)^{k}}{k !} \exp \left(-\gamma A_{\mathrm{Sa}}\right) .
$$

Clearly, the Poissonian process does not fit the data, indicating that the HS are not uniformly distributed over all possible pixels. Instead, they are distributed among some specific sites. We now investigate the distribution of HS among the $M^{\prime}$ sites. Figures 6(b), 6(d), and 6(f) show the probability of having $N$ resonances on a given site. It is seen that the probability is exponential and hardly depends on the filling 

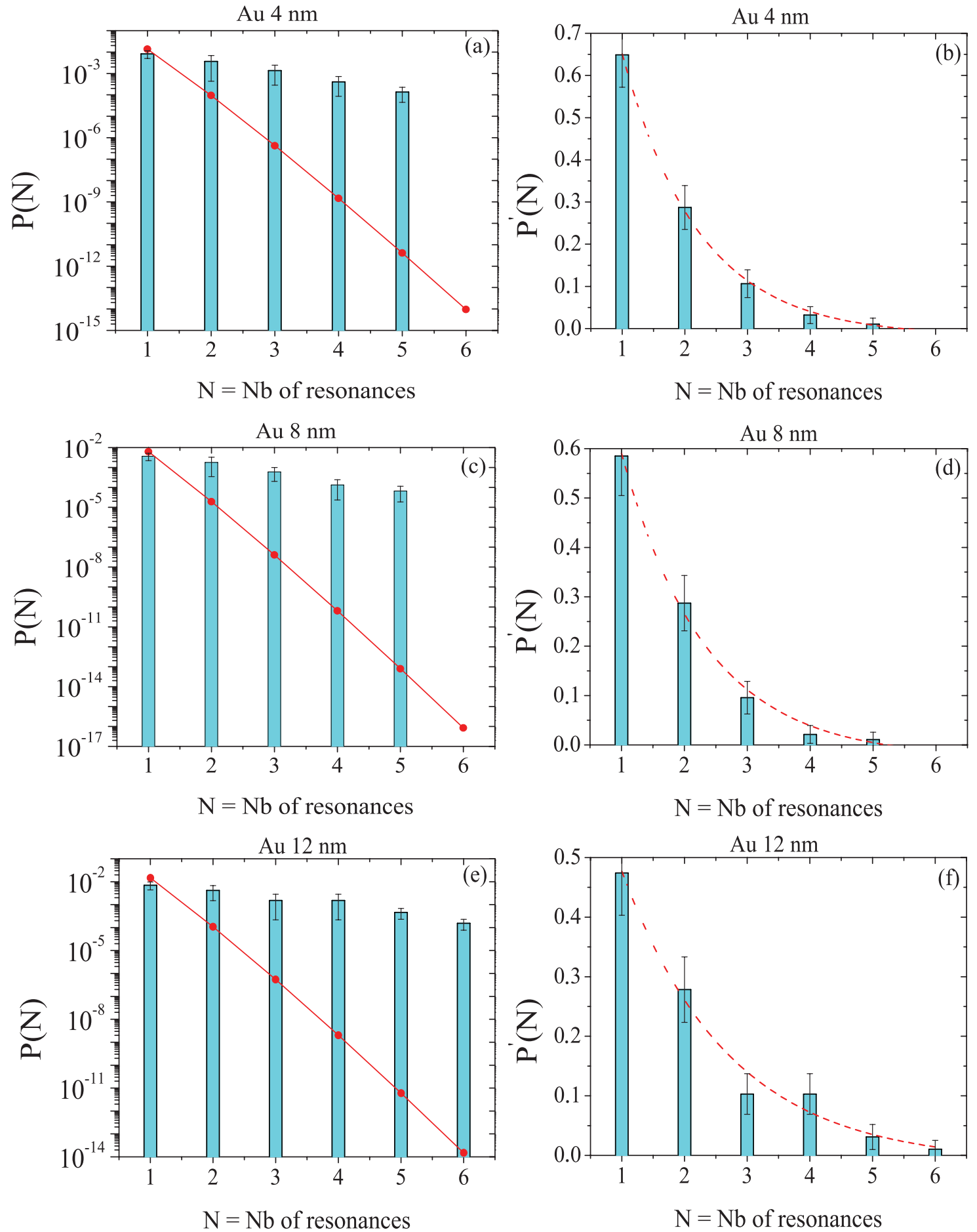

FIG. 6. (Color online) Statistical analysis of the distribution of HS among the pixels. Panels (a), (c), and (e) show $P(N)$, the probability to observe $N$ resonances on a pixel. The red line is the Poisson law. Panels (b), (d), and (f) show $P^{\prime}(N)$, the probability to observe $N$ resonances in a site. The dashed red line is the exponential law.

fraction of the surface. To our knowledge, this exponential distribution had not been reported previously.

\section{CONCLUSIONS}

In this paper, the statistical intensity distributions of the HS characteristic of gold/glass films under light excitation were measured by photoemission electron microscopy in the
800- to $1040-\mathrm{nm}$ range by spectral steps of $10 \mathrm{~nm}$ and a $20-\mathrm{nm}$ spatial resolution. This imaging technique gives backgroundfree images with an excellent accuracy without the perturbative interactions of a SNOM tip. The statistical distributions, density of HS, and 3D PEEM images confirm the predictions of the scaling theory in the near-infrared region. We further find that the statistical distribution of spot intensities follows a log-normal law. We have observed in particular a long tail 
distribution for large intensities close to the percolation threshold. An analysis of the spatial distribution of the HS shows that HS appearing at different frequencies are preferentially located on some specific sites of the semicontinuous film and that the probability of observing $N$ resonances on a given site at different frequencies decays exponentially with $N$.

\section{ACKNOWLEDGMENTS}

We acknowledge financial support by the Agence Nationale de la Recherche (ANR) in the frame of its program in nanosciences and nanotechnologies (PEEMPlasmon Contract No. ANR-08-NANO-034) and by the Triangle de la physique (Contract No. 2008-057T). *jean-jacques.greffet@institutoptique.fr

${ }^{1}$ C. G. Granqvist and O. Hunderi, Phys. Rev. B 16, 3513 (1977).

${ }^{2}$ U. Kreibig and M. Vollmer, Optical Properties of Metal Clusters (Springer, Berlin, 1995).

${ }^{3}$ V. M. Shalaev, Nonlinear Optics of Random Media (Springer, Berlin, 2000).

${ }^{4}$ M. I. Stockman, L. N. Pandey, L. S. Muratov, and T. F. George, Phys. Rev. Lett. 72, 2486 (1994).

${ }^{5}$ M. I. Stockman, L. N. Pandey, L. S. Muratov, and T. F. George, Phys. Rev. Lett. 75, 2450 (1995).

${ }^{6}$ V. M. Shalaev and A. K. Sarychev, Phys. Rev. B 57, 13265 (1998).

${ }^{7}$ D. A. Genov, A. K. Sarychev, and V. M. Shalaev, Phys. Rev. E 67, 056611 (2003).

${ }^{8}$ M. I. Stockman, Phys. Today 64, 39 (2011).

${ }^{9}$ M. I. Stockman, S. V. Faleev, and D. J. Bergman, Phys. Rev. Lett. 87, 167401 (2001).

${ }^{10}$ D. P. Tsai, J. Kovacs, Z. Wang, M. Moskovits, V. M. Shalaev, J. S. Suh, and R. Botet, Phys. Rev. Lett. 72, 4149 (1994).

${ }^{11}$ S. Gresillon, L. Aigouy, A. C. Boccara, J. C. Rivoal, X. Quelin, C. Desmarest, P. Gadenne, V. A. Shubin, A. K. Sarychev, and V. M. Shalaev, Phys. Rev. Lett. 82, 4520 (1999).

${ }^{12}$ S. I. Bozhevolnyi, V. A. Markel, V. Coello, W. Kim, and V. M. Shalaev, Phys. Rev. B 58, 11441 (1998).

${ }^{13}$ S. Ducourtieux, V. A. Podolskiy, S. Grésillon, S. Buil, B. Berini, P. Gadenne, A. C. Boccara, J. C. Rivoal, W. D. Bragg, K. Banerjee, V. P. Safonov, V. P. Drachev, Z. C. Ying, A. K. Sarychev, and V. M. Shalaev, Phys. Rev. B 64, 165403 (2001).

${ }^{14} \mathrm{~S}$. Buil, J. Aubineau, J. Laverdant, and X. Quélin, J. Appl. Phys. 100, 063530 (2006).

${ }^{15}$ K. Seal, D. A. Genov, A. K. Sarychev, H. Noh, V. M. Shalaev, Z. C. Ying, X. Zhang, and H. Cao, Phys. Rev. Lett. 97, 206103 (2006).
${ }^{16}$ V. Krachmalnicoff, E. Castanie, Y. DeWilde, and R. Carminati, Phys. Rev. Lett. 105, 183901 (2010).

${ }^{17}$ C. Wiemann, D. Bayer, M. Rohmer, M. Aeschlimann, and M. Bauer, Surf. Sci. 601, 4714 (2007).

${ }^{18}$ L. Douillard, F. Charra, C. Fiorini, P. M. Adam, R. Bachelot, S. Kostcheev, G. Lerondel, M. Lamy de la Chapelle, and P. Royer, J. Appl. Phys. 101, 083518 (2007).

${ }^{19}$ H. Kawano, Prog. Surf. Sci. 83, 1 (2008).

${ }^{20}$ G. P. Banfi, G. Ferrini, M. Peloi, and F. Parmigiani, Phys. Rev. B 67, 035428 (2003)

${ }^{21}$ A. Gloskovskii, D. A. Valdaitsev, M. Cinchetti, S. A. Nepijko, J. Lange, M. Aeschlimann, M. Bauer, M. Klimenkov, L. V. Viduta, P. M. Tomchuk, and G. Schönhense, Phys. Rev. B 77, 195427 (2008).

${ }^{22}$ W. Pfeiffer, C. Kennerknecht, and M. Merschdorf, Appl. Phys. A 78, 1011 (2004).

${ }^{23}$ L. Douillard, F. Charra, Z. Korczak, R. Bachelot, S. Kostcheev, G. Lerondel, P.-M. Adam, and P. Royer, Nano Lett. 8, 935 (2008).

${ }^{24}$ C. Hrelescu, T. K. Sau, A. L. Rogach, F. Jäckel, G. Laurent, L. Douillard, and F. Charra, Nano Lett. 11, 402 (2011).

${ }^{25}$ J. A. Quintanilla and R. M. Ziff, Phys. Rev. E 76, 051115 (2007).

${ }^{26}$ K. Seal, A. K. Sarychev, H. Noh, D. A. Genov, A. Yamilov, V. M. Shalaev, Z. C. Ying, and H. Cao, Phys. Rev. Lett. 94, 226101 (2005).

${ }^{27}$ M. L. Brongersma, J. W. Hartman, and H. A. Atwater, Phys. Rev. B 62, R16356 (2000).

${ }^{28}$ A. L. Burin, H. Cao, G. C. Schatz, and M. A. Ratner, J. Opt. Soc. Am. B 21, 121 (2004).

${ }^{29}$ A. K. Sarychev and V. M. Shalaev, Phys. Rep. 335, 275 (2000).

${ }^{30}$ R. C. Word, T. Dornan, and R. Konenkamp, Appl. Phys. Lett. 96, 251110 (2011) 